\title{
Redefining Adult Cosmetic Orthodontics
}

\author{
Clift Georgaklis* \\ Cleveland Circle Dental Associates, 1798 Beacon St, Brookline, Massachusetts, US
}

Received: April 08, 2015; Accepted: July 09, 2015; Published: July 17, 2015

*Corresponding author: Clift Georgaklis, Cleveland Circle Dental Associates, 1798 Beacon St, Brookline, Massachusetts, US, E-Mail: cgeorgaklis@comcast.net

\section{Background and History}

In the early 1900's, Edward Angle was a pioneer that lead orthodontics into becoming its own speciality. He founded the first school of orthodontics, the American Society of Orthodontia in 1901, and the first orthodontic journal in 1907. As the "father of modern orthodontia", he heavily influenced treatment towards establishment of an Angle Class I occlusion, classifying malocclusions as Class I, II, and III based on molar occlusion. Yet the meaningfulness of this classification system is often brought into question [1]. There is less disparity among orthodontists when classifying occlusions according to incisal classifications of overjet and overbite, as many do in Britain [2]. There has yet to be definitive evidence in the literature to support lengthening treatment to establish a Class I molar occlusion if it is otherwise functional.

When the popularity of orthodontic treatment surged in the 1950's, esthetic dentistry had not yet come into its own. America's dental awareness and cosmetic expectations were low. With the advent of acid etching, cosmetic bonding, and later porcelain veneers, the practice of cosmetic dentistry progressed. Slowly more adults began to undergo orthodontic treatment also. They usually wanted straight teeth and a pretty smile. Over time, orthodontists began to notice that the general public wanted straight teeth and was less concerned with the type of occlusion and cephalometric measurements than they were [3], as long as they functioned without attrition, open bite, periodontal loss, or other health related issues.

Bonding brackets instead of banding made it possible to judiciously make space locally through the interproximal reduction of enamel surfaces, popularized by Dr Jack Sheridan [4]. It has taken decades for enamel reproximation to become more accepted, and now it is widely used over the all or none measure of bicuspid extraction, which requires closing a greater amount of space made far from the crowded incisors. Single lower incisor extraction also came into use in the 1970's [5] and 1980's.

\section{Let's Try Something Different}

After learning about these two simple less invasive ways of making space, I was instantly hooked. Brainwashed by long term treatment dogma in dental school, I was convinced I was doing something wrong, or just missing something. The potential to shorten treatment was readily apparent as I began my first cases in general private practice in 1991. As the teeth straightened quickly by creating space locally, as well as through nickel titanium wire use, patients were asking why the braces needed to be on longer. As they generally had begun with well functioning occlusions, I had no answer. My orthodontic treatment times were reduced with limited occlusal change (treating crossbites, anterior deep bites, and overjet reduction) to 6 months or less when I wasn't extracting bicuspids- which was done rarely. Through the use of fixed anterior composite bite planes behind the incisors, deep bites corrected quickly through passive supraeruption of molars, and some intrusion of the incisors. I started treating a wide variety of cases and occlusions in an ethnically diverse city. I found that resolving incisal Class III occlusions and posterior cross-bites usually could also be treated in a short time frame. Although not all of my patients have finished with a Class I molar occlusion over the last 24 years, they function well over time at recalls, just like molar Class I cases. Bruxers experienced attrition with whatever type of occlusal guidance or molar occlusion they had. It was obvious they needed a nightguard, rather than choosing which teeth they would wear down and have abfractions on. I could not discern any differences in stability or attrition at recalls between patients finished with a molar Class I, II, or III. "Less than $15 \%$ of the population develops a normal occlusion defined by Angle in the permanent dentition. The term 'ideal' may therefore be a more appropriate description, and deviations from this esthetic and functional optimum should not be considered abnormalities in the true sense of the word. Current research indicates that few malocclusions compromise dental, periodontal or temporomandibular health" [6].

Treatment of TMD is another controversial area in which we have used long term bite changing orthodontics. Many claim TMD improvement and the cessation of bruxism during their orthodontic treatment as proof of successful occlusal rehabilitation, and therefore predictive of long term joint health. Given the sparseness of research supporting this, the more likely reason for improvement is teeth are too sore to be clenching during active treatment. Though I never promise TMD resolution in performing orthodontics, I have had remarkable long term success in TMD cases through avoiding retrusive incisor contact. Establishing liberal overjet avoids retrusive anterior contact and disc impingement, even if there is relapse or non-compliance with retention. After numerous studies, comprehensive 2 year orthodontic treatment has not been proven to improve TMD [7]. 
Those who claim long-term successful resolving TMD through orthodontic treatment would do well to put forth more definitive research supporting it.

So from 1991 - 1999 in my general practice, with a cosmetically focused treatment approach I discovered that the majority of adult cosmetic orthodontic cases could be aligned in 6 months time or less having treated hundreds of cases, and decided to write about it (Georgaklis, 1999). Many of these cases had significant crowding and were finished with a highly cosmetic result that excited the patients and myself.
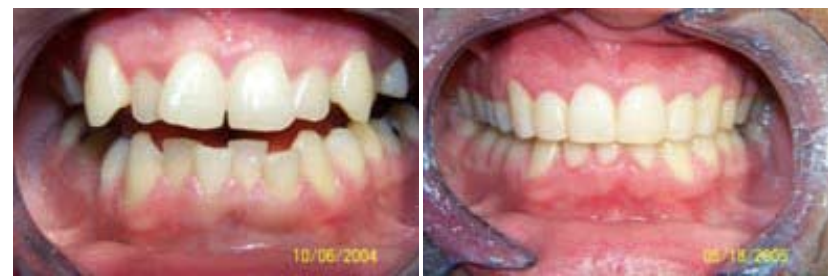

An example of the typical case we see for adult accelerated orthodontics (AAO) with upper and lower splinting canine to canine for reliable retention. We recommend the splints stay on for a minimum of 5 years before sequential removal.

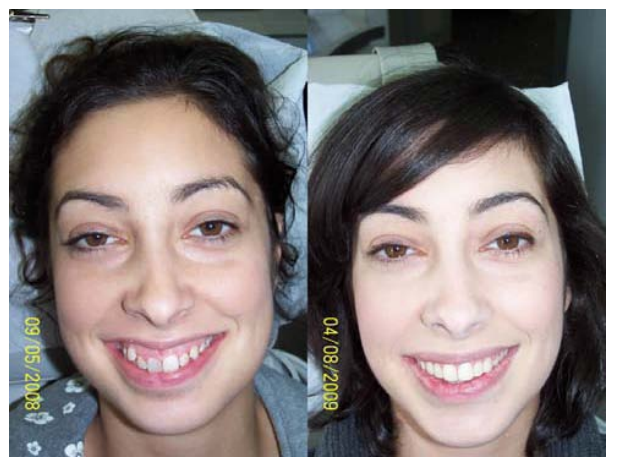

Many principles of smile design are incorporated into adult accelerated treatment (AAO) beyond alignment such as decreasing gingival display and addressing tooth proportions, shape, and color.

These cases are also stable by avoiding posterior lateral expansion, maintaining arch circumference and functional posterior occlusion. There are very few tooth movements (of erupted anterior teeth) that take greater than 6 months to align if space can be made without bicuspid extraction. I used to think that shorter treatment times would bring more root resorption, based on the assumption that greater force was used. But greater force is not what makes shorter treatment time possible, but that space is made locally near the crowding. There is less tooth movement, less bone remodeling, less inflammation, and less time for the roots to resorb, if the individual is prone to this distinctive hyper inflammatory response. It is longer treatment times using extractions that has been repeatedly and conclusively proven to be the cause of significant root resorption [8].

The Age of Accelerated Adult Orthodontics (AAO)

After practicing AAO for 24 years, I believe that avoiding difficult and lengthy adult molar translational movements that are nearly impossible to retain (in an attempt to obtain a molar Class I) is truly beneficial for the patient. "Minimally invasive cosmetic dentistry" as Maini [9] describes adult aesthetic orthodontics, can be an effective tool for adults with a cosmetic chief complaint. As many practitioners of AAO know, the three most important reasons for treatment are "aesthetics, aesthetics, and aesthetics" [10]. Perhaps the acronym should include ADULT ACCELERATED AESTHETIC ORTHODONTICS and be AAAO. So far everyone has their label. But everyone knows treatment is accelerated for aesthetic reasons, so the word "aesthetic" is redundant. Many patients are coming back to the dentist for this service who has been avoiding us. $2 / 3$ of them in my practice have frank caries and other restorative and periodontal issues. AAO is a more appropriate and conservative treatment than some the most well intentioned restorative dentistry replacing enamel and dentin with porcelain or composite in "restoring smile harmony" [11].

Although AAO is not yet standardized, the simplified approach is growing [12]. The foundational thinking is listening to the patients chief complaint and treating it, suggesting (but not deciding for them) what they must endure to get the smile they came for. Suggesting excessive overjet reduction when it exists is necessary, changing molar occlusion can be very helpful (especially with crossbites), but insisting on both across the board can legitimately be considered overtreatment that I would not want for myself or my children. As Mohlin [13] said on malocclusions and aesthetic treatment, "The mere presence of deviations from the concept of the ideal occlusion should have no influence on orthodontic treatment decisions. According to studies, the influence of malocclusion on periodontal health, speech and chewing is fairly minor. Neither can orthodontic treatment be justified as an effective means of preventing TMD but it may be indicated to reduce the existing signs and symptoms of TMD in certain carefully selected cases. Interceptive or preventive orthodontic treatment may be indicated to reduce the negative influence on growth and occlusal development of functional malocclusions (anterior or lateral forced bite) or ectopic tooth eruption. Similarly, early correction of large overjet may be valuable in order to reduce the risk of traumatic injuries. Such treatment is usually motivated during the primary or mixed dentition periods. From the teenage period onwards, psychosocial or aesthetic reasons for orthodontic treatment are dominating. Decisions to start orthodontic treatment in order to improve aesthetics should usually not be taken before the child has reached sufficient maturity for these decisions, normally after the age of 12 years." This well summarizes my treatment approach on AAO, pediatric treatment, and TMD orthodontics.

Since the arrival of Invisalign, which was specifically formulated for anterior alignment in the adult cosmetic patient about 15 years ago, the limited treatment concept does not seem so controversial. Perhaps this is because the method of delivery is less invasive with removable appliances. But when I began marketing cosmetic orthodontics in 1991-1992, it was considered 
radical. With AAO, straight teeth are just a starting point though. There are various other modalities that are incorporated into treatment that haven't yet been because of the narrow occlusal focus of the past. Teeth can be straight but appear unaesthetic.
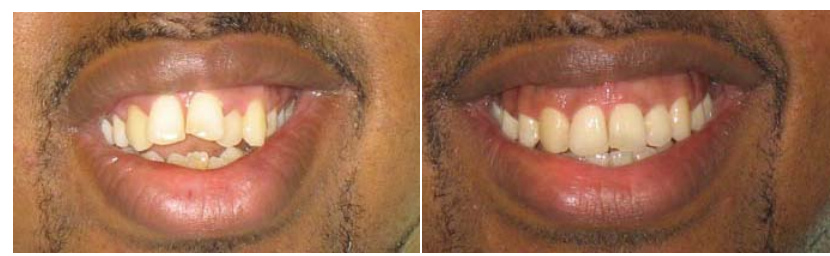

Treatment in the above case shows lack of attention to anterior tooth morphology, incisal congruence, stepping, and proportion, gingival display and height, and color- despite proper alignment and open bite closure. The final result also ignores the gingival and anatomical accommodations needed on the right side for canine substitution.

\section{So what is different about AAO?}

Accelerated treatment time and lingual orthodontics: We know that long treatment time is one of the greatest barriers to orthodontic treatment. Though thermally activated, nickeltitanium wire use, limited occlusal change, enamel reproximation, lower incisor extraction, and to some extent lingual orthodontics, you would have to live under a rock not to realize that adult orthodontics has undergone a rebirth [14]. Any arch expansion treatment past the age of alveolar growth (usually 12-14 years old) will be more prone to relapse [15], so we avoid expansion and bicuspid extraction when possible. In cases with significant crowding or spacing I suggest canine to canine splinting. Posterior cross bite correction in adults requires permanent removable retainer wear at night. While it is far more difficult to control tooth movements with lingual orthodontics, it is another option that eliminates objections to treatment. It is far easier to perform lingual orthodontics when focusing on the anteriors and the occlusion is maintained.

Fine tuning proportion: In my office, interproximal reduction is performed with Brasseler diamond discs of $.15 \mathrm{~mm}$ thickness, so very little enamel is removed, even if the same contact is disked on multiple visits. After measuring the mesialdistal dimensions of disked anterior teeth on castes before and after treatment in multiple cases, I could not detect the difference because it was so small. When making space on multiple visits, wide teeth are disked to maintain symmetry. Incisal edges can also be adjusted to maintain proportion.
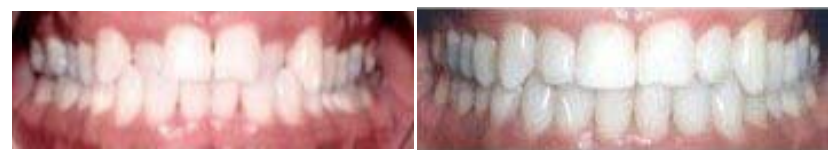

This excellent orthodontic result could have been made better through enamelplasty.

We re-establish proportionate interproximal and incisal embrasures so teeth maintain their shape.

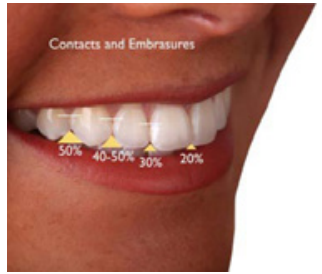

Teeth are "shrunken" in every dimension, not just interproximally. No one should be able to detect where enamel was removed.
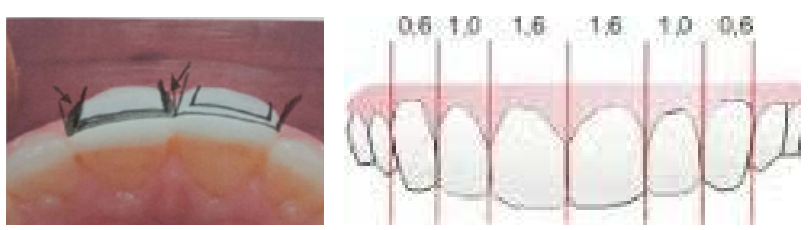

The positioning of facial line angles also changes perceived width as shown on the left. Using high magnification we can modify facial-interproximal line angles to change the apparent widths of facial surfaces so they relate to other teeth in the Golden Proportion shown above right.
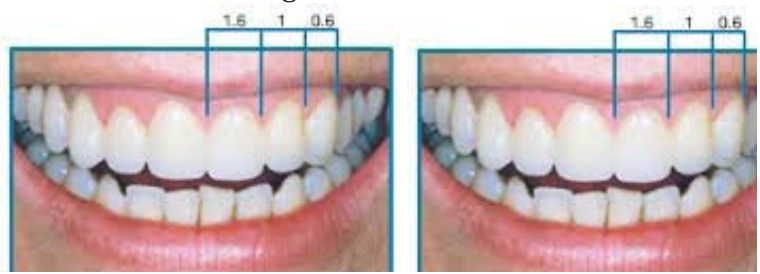

Although both smiles show the Golden Proportion in widths relative to other teeth in the arch, the length to width ratio on the right simulation is more appealing. Shortening anterior teeth on the left dentition would provide better proportion.

Narrow teeth such as peg lateral incisors are bonded before space closure. I reshape incisal edges noting proper offsets, and facial surfaces to change apparent root angulations. I bond or bleach uneven, chipped, fluorosed, decalcified, or dark teeth, and establish proper gingival display of $0-2 \mathrm{~mm}$ [16] through gingival surgery. If the deficient dimension of the tooth is on the incisal due to attrition, bonding or porcelain is added. While thin incisal composites normally fracture, the thickness is greater when supported by a lingual splint and remarkably durable.

We can deliver a white, wide, full arch smile that is retained where necessary through splinting, in a timely manner in the vast majority of cases. Thoughtful delivery of finishing procedures according to each patient delivers the ideal result.

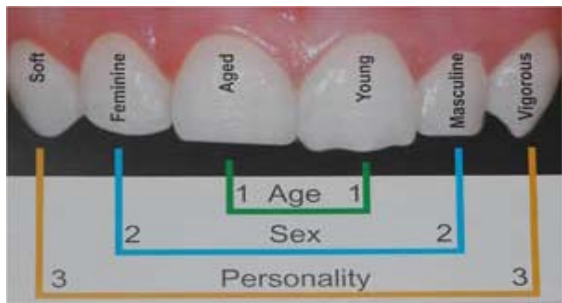

Tooth morphology should be custom. Unless the patient specifies otherwise, I prefer the rounded incisor appearance. 

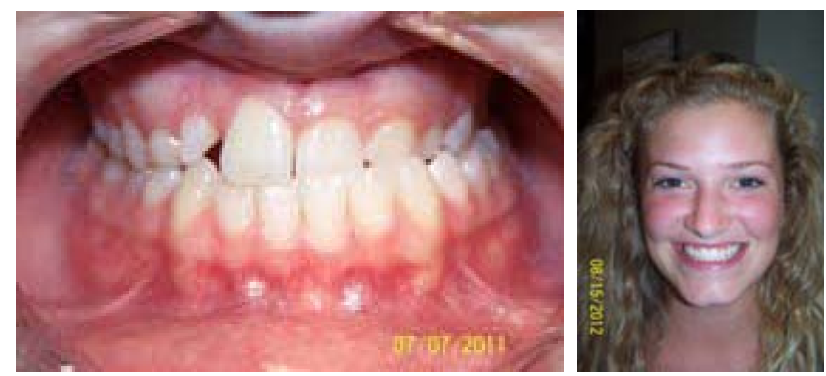

While orthodontics leveled the plane of occlusion, corrected the cross bite, and positioned roots, porcelain was needed to address the peg lateral on the right, canine substitution on the left, and replacement of both mobile primary canines which were extracted.
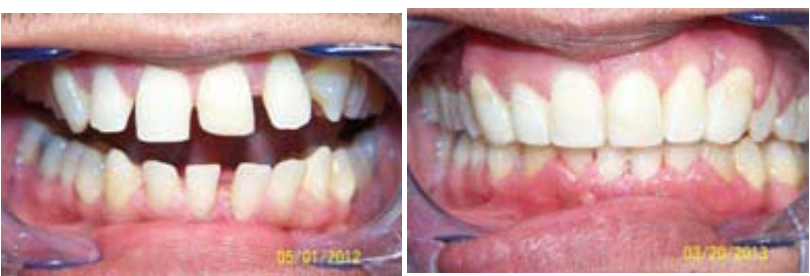

Acid erosion diminished incisor widths and congruence, especially in the incisal third. After orthodontics a composite splint retained the result and added proportion, without extensive removal of enamel for crowns. Thin sections of composite match well and can be hard to detect.
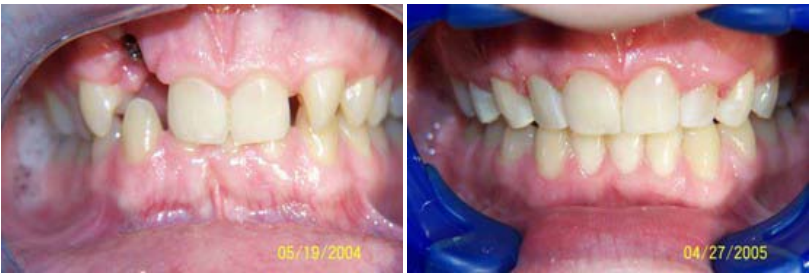

This involved case demonstrates the manipulation of line angles, incisal edges, and gingival contour essential when performing canine substitution for missing upper lateral incisors. After the impacted upper right canine was extruded, both canines were reshaped, bonded, and made into a lateral incisor. Deep bite correction was achieved through a fixed flat composite bite plane lingual to 8 and 9. Moving the canine facial-interproximal line angle to the center of the tooth narrows the facial aspect, turning each canine into a lateral. Shortening the point, flattening the face, and adding incisal corners hides the cylindrical qualities of the canine. Bicuspids were flattened facially on the incisal third to resemble canines- anatomical hybrids of incisors and bicuspids.

Soft tissue aesthetics and congruence: Gingival contour and position can often be manipulated because many cases in the younger population undergoing AAO and cosmetic dentistry require removal of gingiva to establish congruence more than addition. Most patients with high smile lines do not have concurrent recession problems due to the thick bone and gingiva, as shown below.

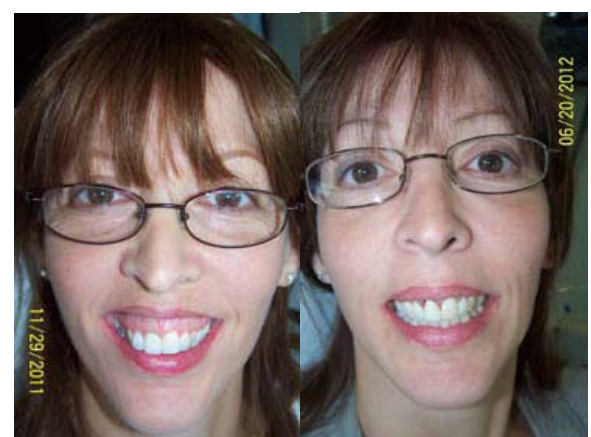

This case was treated through intrusion of the maxillary incisors in conjunction with successive gingivectomies. A periodontal pocket was re-established to maintain biologic width and minimize regrowth of gingiva post treatment. Photo on right was after emax crown cementation on \#8 with the lingual splint bonded directly to the tooth.

If the gingival level after surgery does not allow for biologic width defined as the space between alveolar bone attachment and the free gingiva margin, the gingiva will regrow to re-establish a protective zone for the bone. After performing a gingivectomy I use a narrow fluted burr to make a shallow pocket of 1-2 mm severing the crested periodontal attachment (an "augmented gingivectomy") to prevent regrowth of the gingiva back to its previous level. This limits regrowth of gingival.
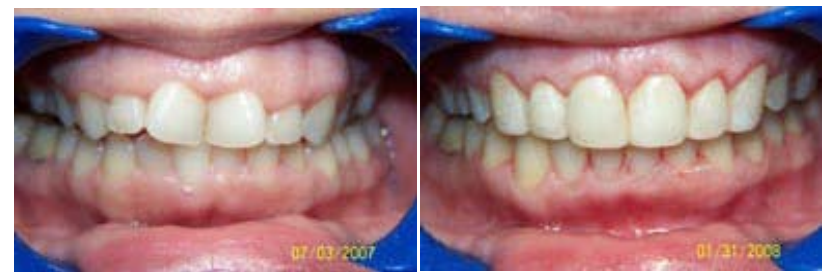

After AAO, and 2 days after augmented gingivectomy.

As moving teeth together for space closure can constrict, enlarge, and lengthen the papillae, deficient papillae can be made to fill black triangular spaces. Overgrown swollen papillae should be trimmed as they collect more plaque and are unaesthetic. Triangular shaped teeth can have recession of the papillae because the contact point is more incisal and the bone crest is more apical which results in a flatter papilla [17]. A flatter contact after reproximation usually elongates the papilla so that it is "pinched", and often able to fill the black triangular space though. This commonly occurs in space closure. Many times I treat black triangular spaces in this way, saving the patient an involved periodontal surgical procedure.

Brandao [18], an orthodontist in one of the most esthetically conscious countries in the world (Brazil) well described finishing procedures in orthodontics; "Treatment quality is directly related to the amount of procedures implemented by the orthodontist, associated with concepts and resources from Periodontics and Dental Prosthesis. Microesthetics cannot be seen in isolation, but rather as the key to establish a pleasant smile (miniesthetics) in addition to a harmonious face (macroesthetics) and a human 
being with high self esteem (hyper-esthetics)." Occlusion is no longer the sole focus of orthodontics, and that the "attainment of perfect occlusal results does not ensure stability" [19]. Many know that the "ideal posterior occlusion concepts as a general orthodontic treatment goal should be reconsidered" [20]. Patients can tell their type of incisor occlusion, but it does not correlate with molar occlusion. Posterior occlusion is difficult to maintain, and even mandibular incisor relapse has been termed "inevitable" [21]. Any tooth movement when done past the stage of growth and alveolar arch adaptation is more likely to relapse [15], especially expansion of the intercanine width [22].

Fortunately anterior teeth that have been moved can be splinted. Expecting the majority of the adult population to reliably wear removable retainers permanently (as textbooks say) is unrealistic. For any significant anterior tooth movement, upper or lower, I strongly suggest a bonded splint. Anterior fixed retention prevents the "inevitable" incisor rotations while still allowing posterior settling [23]. Once teeth are straight patients focus on other dental imperfections, so identifying aesthetic restorative and periodontal treatments should be part of the plan.

\section{Conclusion}

The patient should understand the full scope of the problem and treatment plan at the initial consult to avoid disappointment by undergoing orthodontics alone. Few orthodontic practitioners address comprehensive dental esthetics because of the different skill sets involved. Ultimately we seek to deliver straight, white teeth, with symmetry, proportion, lip support, minimal gingival display, attractive facial embrasures, and a durable result, in an efficient treatment time, that the patient is happy with years later. It is thrilling to hear a patient say it was the best thing they ever did for themselves.

A brilliant smile is a puzzle integrating various small parts. Cosmetic dentistry is long past focusing only on whitening (although some endodontically treated dark incisor roots can still be hard to opaque from light refraction coronally). Putting the pieces together for a spectacular lasting result is within our grasp for the majority of cases, thanks to the evolution of materials, techniques, experience, and creative thinking.

\section{References}

1. Brin I, Weinberger T, Ben- Chorin E. Classification of occlusion reconsidered. The European Journal of Orthodontics. 1999;22(2):169174. doi: 10.1093/ejo/22.2.169.

2. Williams AC, Stephens CD. A modification to the incisor classification of malocclusion. Br J Orthod. 1992;19(2): 127-130.

3. Cochrane SM, Cunningham SJ, Hunt NP. Perceptions of facial appearance by orthodontists and the general public. J Clin Ortho. 1997;31(3):164-168

4. Sheridan JJ. Air rotor stripping. J Clin Orthod. 1985;19(1):43-59.

5. Rosenstein SW. A lower incisor extraction?. Aust Orthod J. 1976;4(3):107-109.

6. Artun J. The role of minimal intervention in orthodontics. Med Princ

\section{Pract. 2002;11(1):7-15}

7. Luther F, Layton S, McDonald F. Orthodontics for treating temporomandibular joint (TMJ) disorders. Cochrane Database Syst Rev. 2010;7:CD006541.doi: 10.1002/14651858.

8. McFadden WM, Engstrom C, Engstrom H, Anholm JM. A study of the relationship between incisor intrusion and root shortening. Am J Orthod Dentafacial Orthop. 1989;96(5):390-396.

9. Maini A. Short term orthodontics for general dental practitioners. Br Dent J. 2013;214(2):83-84.doi: 10.1038/sj.bdj.2013.52.

10. Cerny R. What are the three most important reasons for orthodontics treatment? Are they aesthetics, aesthetics, and aesthetics? Aust Orthod J. 2005;21(2):156-160.

11. Prieto LT, Araujo CT, de Oliveira DC, de Azevedo Vaz SL, D’Arce MB, Paulillo LA. Minimally invasive cosmetic dentistry: smile reconstruction using direct resin bonding. Gen Dent. 2014;62(1):2831.

12. Noar JH, Sharma S, Roberts-Harry D, Qureshi T. A discerning approach to simple aesthetic orthodontics. Br Dent J. 2015;218(3):157-166. doi:10.1038/sj.bdj.2015.55.

13. Mohlin B. To what extent do deviations from an ideal occlusion constitute a health risk? Swed Dent J. 2003; 27(1):1-10.

14. Hutchinson I. Lee JY. Fabrication of lingual orthodontic appliances: past, present and future. J Orthod. 2013;40 (1):14-19.doi:10.1179/ ortho/40.s1.s14.

15. Bishara SE, Jakobsen JR, Treder J, Nowak A. Arch Width changes from 6 weeks to 45 years of age, Am J Orthod Dentofacial Orthop. 1997;111(4):401-409.

16. Akhare PJ, Daga A. Effect of the gingival display on posed smile with different facial forms: a comparison of dentists and patients concepts. Indian J Dent Res. 2012;23(5):568-573.doi:10.4103/09709290.107328

17. Kim JH, Cho YJ, Lee JY, Kim SJ, Choi JI. An analysis on the factors responsible for relative position of interproximal papilla in healthy subjects. J Periodontal Implant Sci. 2013;43(4):160-167.doi:10.5051/ jpis.2013.43.4.160.

18. Brandao RC, Brandao LB. Finishing procedures in orthodontics: dental dimensions and proportions (microesthetics). Dental Press J Orthod. 2013;18(5):147-174.

19. Nett BC, Huang GJ. Long term posttreament changes measured by the American Board of Orthodontics objective grading system. Am J Orthod Dentofacial Orthop. 2005127(4):444-450.

20. Knosel M, Jung K. On the relevance of "ideal”occlusion concepts for incisor inclination target definition. Am J Orthod Dentofacial Orthop. 2011;140(5):652-659.doi:1016/j.ajodo.2010.12.021.

21. Shah AA. Postretention changes in mandibular crowding: a review of the literature. Am J Orthod Dentafacial Orthop. 2003;124(3):298-308.

22. Fleming PS, Dibiase AT, Lee RT. Arch form and dimensional changes in orthodontics. Prog Orthod. 2008;9(2): 58-64.

23. Aszkler RM, Preston CB, Saltaji H, Tabbaa S. Long term occlusal changes assessed by the American Board of Orthodontics' model grading system. Am J Orthod Dentofacial Orthop. 2014;145(2):173178. doi:10.1016/j.ajodo.2013.10.010. 